\section{Digitales Recht oder digitale Gerechtigkeit?}

\author{
von Susanne Giesecke und David Linse, \\ VDI/VDE-Technologiezentrum Information- \\ stechnik
}

Die unter dem Schlagwort "Digital Divide“" geführt Debatte impliziert den uneingeschränkten Zugang aller Menschen zum Internet und der in ihm verfügbaren Informationen zu jeder Zeit. Viele Aspekte werden jedoch dabei außer Acht gelassen: z. B. die oft mangelnde Nutzerkompetenz oder gesellschaftliche Randgruppen, die auch in den Initiativen zur Überwindung der digitalen Kluft nicht integriert werden. Mit der zunehmenden Etablierung von Property Rights Regimen wächst zudem parallel die Gefahr einer weiteren Zementierung der digitalen Kluft. Statt durch den Einsatz neuer luKMedien gesellschaftliche Probleme zu lösen, werden neuen Fronten geschaffen.

\section{Der Diskurs über das Phänomen Digital Divide}

Der Terminus „Digital Divide“, im Deutschen auch als Digitale Kluft oder Digitale Spaltung übersetzt, erfreute sich in jüngster Vergangenheit zunehmender Beliebtheit und avancierte geradezu zum Modebegriff. Die Vielzahl einschlägiger Publikationen und Erklärungen hat längst ein Ausmaß erreicht, das es unmöglich macht, diesen Bestand in Gänze zu erfassen. Neben diversen sozial- und wirtschaftswissenschaftlichen Beiträgen lassen sich hierbei v. a. Stellungnahmen von Medien, Verbänden und diversen Initiativen ausmachen. Auch in den TA-Datenbank-Nachrichten wurde die Thematik an verschiedenen Stellen angedeutet - zumeist wurde Digital Divide hierbei als Hemmschuh im Hinblick auf die Entwicklung des elektronischen Handels analysiert (vgl. bspw. Hauser 2001, S. 4; Hochreiter 2001, S. 6).

Digital Divide wird dabei zumeist im Kontext der entstehenden Wissens- bzw. Informationsgesellschaft thematisiert, einer Gesellschaftsform, in der mittels Wissensarbeit ein überwiegender Teil des Sozialproduktes erwirtschaftet wird und in der alle Individuen auf den freien Zugang zu Wissen angewiesen sind, um sich sozial und ökonomisch entfalten zu können (Kuhlen 2001, S. 2f.). Dementsprechend wird ein umfassender Einsatz von Informations- und Kommunikationstechnologien - den elementaren „Werkzeugen“ der Informationsgesellschaft - auch als grundlegende Voraussetzung für nationale Wettbewerbsfähigkeit und ökonomische Prosperität betrachtet. So formuliert die „Initiative D21“61, die nach eigenen Angaben den Wandel Deutschlands von der Industrie- zur Informationsgesellschaft beschleunigen möchte, in ihrem ,Memorandum“:
„Das Informationszeitalter hat unwiderruf- lich begonnen. Die weltweite information- stechnische Vernetzung gibt diesem Zeitalter seine globale Dimension. Es gibt daher keine nationalen Schutzzonen mehr. Die aktuelle und aktive Nutzung von moderner Informa- tions- und Kommunikationstechnik in allen Bereichen unserer Gesellschaft entscheidet über viele neue und dauerhafte Arbeitsplätze sowie nachhaltigen Wohlstand" (Initiative D21 1999).

Gleichwohl wird Kompetenz im Umgang mit IuK-Technologien im Allgemeinen und dem Internet im Besonderen nicht nur als relevant für die Entwicklung nationaler Ökonomien erachtet. „Schulen ans Netz“2 ${ }^{\text {, }}$ eine gemeinsame Initiative des Bundesministeriums für Bildung und Forschung sowie der Deutschen Telekom AG, hat sich die Förderung des Interneteinsatzes in Schulen zum Ziel gesetzt und erklärt den Umgang mit dem World Wide Web und komplementären Technologien somit implizit zum Gegenstand der Allgemeinbildung, der für die individuelle Persönlichkeitsentwicklung unverzichtbar geworden ist.

Die entsprechenden Initiativen und Stellungnahmen transportieren denn auch die Botschaft, dass der allgemeine Zugang zu Wissen - und dies bedeutet in der Informationsgesellschaft v. a. Zugang zu IuK-Technologien eine Grundvoraussetzung ist, um in vollem Umfang am sozialen und ökonomischen Leben partizipieren zu können. Staatsrechtlich untermauert wird diese Einschätzung nicht zuletzt durch Artikel 5 (GG):

(1) Jeder hat das Recht, seine Meinung in Wort, Schrift und Bild frei zu äußern und zu verbreiten und sich aus allgemein zugänglichen Quellen zu unterrichten. ${ }^{3}$

Als empirische Legitimationsbasis ziehen die entsprechenden Maßnahmen und Initiativen eine Reihe von Primärerhebungen heran, die seit 
mehreren Jahren periodisch die Verteilung von Internetanschlüssen in der Bevölkerung und/ oder die Soziodemographie der Internetnutzer erheben. Die Trennlinien zwischen „Onlinern“ und „Offlinern“ verlaufen demnach entlang der Kategorien Geschlecht, Alter, formalem Bildungsgrad sowie Einkommen. Nach neuesten Erhebungen nutzen derzeit rund $44 \%$ der Deutschen das Internet. Von diesen sind $57 \%$ männlichen Geschlechts, aber nur $43 \%$ Frauen, $65 \%$ sind jünger als 40 Jahre, aber nur $35 \%$ sind 40 Jahre und älter (van Eimeren, Gerhard 2002, S. 348f.). Untersucht man des Weiteren die Kategorien formaler Bildungsgrad sowie Einkommen, lässt sich feststellen, dass $200170 \%$ der Internet-Nutzer über eine mittlere Reife, Abitur und/oder ein abgeschlossenes Hochschulstudium verfügten, während nur $30 \%$ der Onliner als höchsten formalen Bildungsabschluss einen Volks- bzw. Hauptschulabschluss angaben (GfK 2001). Gemessen an der Gesamtbevölkerung besitzen jedoch $44 \%$ eine Volks- bzw. Hauptschulbildung. Die digitale Spaltung verläuft aber nicht zuletzt auch entlang der Kategorie Einkommen. Verfügten $200123 \%$ der deutschen Bevölkerung über ein monatliches Haushaltsnettoeinkommen bis 1.500 Euro, so hatte diese Gruppe unter den Internetnutzern nur einen Anteil von $14 \%$ (ebd.).

Gleichwohl lässt sich in jüngster Vergangenheit eine zunehmende Angleichung der soziodemographischen Strukturen von Gesamtbevölkerung und Internetnutzung aufgrund stärkerer Zuwachsraten der unterrepräsentierten Bevölkerungsgruppen feststellen. So betrug das Verhältnis von Männern und Frauen noch vor vier Jahren 72 zu $28 \%$ und die Relation der bis 40-Jährigen zu älteren Internet-Nutzern betrug 73 zu $27 \%$ (van Eimeren, Gerhard 2002, S. 349). Darüber hinaus muss festgehalten werden, dass die Nichtnutzung moderner IuKTechnologien, und besonders die „Verweigerung" des Internetgebrauchs, nicht mehr nur das Ergebnis sozioökonomischer Ungleichverteilungen, sondern häufig auch Folge einer bewusst gefällten Entscheidung ist. Dafür spricht bspw. die Tatsache, dass die Gruppe der „klassisch Kulturorientierten“ (van Eimeren, Gerhard 2002, S. 349), die tendenziell eher zu den formal Höhergebildeten und Besserverdienenden zu zählen ist, unter den Internetnutzern deutlich unterrepräsentiert ist.
Die vorhandenen Primärerhebungen zur digitalen Spaltung der deutschen Gesellschaft suggerieren einen umfassenden Einblick in die (Ungleich-)Verteilung von Internetzugängen. Dennoch weisen die entsprechenden Untersuchungen eine Reihe von Defiziten auf, die qualitative Aussagen über die Chancen einzelner Bevölkerungsgruppen in der entstehenden Wissensgesellschaft sowie die gesellschaftlichen Folgen des allumfassenden Einsatzes moderner IuK-Technologien erschweren. Um dies $\mathrm{zu}$ verdeutlichen, ist es sinnvoll, den Terminus „Digital Divide“ nochmals präzise zu definieren. Die OECD kommt bei diesem Versuch in ihrer Studie „Understanding the Digital Divi$\mathrm{de}^{\text {" }} \mathrm{zu}$ folgendem Ergebnis:

\section{„The term ,digital divide“ refers to the gap between individuals, households, businesses and geographic areas at different socio- economic levels with regard both to their op- portunities to access information and com- munication technologies (ICTs) and to their use of the Internet for a wide variety of ac- tivities" (OECD 2001, S. 5)}

\section{Defizite der Debatte}

Der Diskurs über die digitale Spaltung in Deutschland verbleibt weitgehend auf der „Hardwareebene“ und reduziert die Analyse der digitalen Spaltung auf die Frage nach den technischen Voraussetzungen zur passivrezeptiven Partizipation. Aus der Definition der OECD wird aber deutlich, dass unter Partizipation an der Wissensgesellschaft vermittels der Verwendung des Internets sowie komplementärer Technologien mehr zu verstehen ist als der bloße technische Zugang. Dies soll im Folgenden an einigen Beispielen erläutert werden.

Die Fähigkeit des Einzelnen, mittels IuKTechnologien an den Möglichkeiten der Wissensgesellschaft partizipieren zu können, hängt nicht zuletzt von bestimmten Kompetenzen ab, die für den Umgang mit diesen Technologien erforderlich sind. Gerade die Nutzung des Internets bzw. des Computers als Medium erfordert zum Teil völlig neue Qualifikationen, die mittels traditioneller Kulturtechniken nicht erlernt werden. Die Tatsache, online zu sein oder virtuos mit dem Computer als Layoutgerät oder Spielmaschine umgehen zu können, sagt noch nichts aus über die Fähigkeiten des Einzelnen, Infor 
mationen mit dem neuen Medium suchen, finden und bewerten zu können (Kubicek 1999). Die bloße Frage nach dem Zugang zum Internet, also die rein technische Seite des Problems der digitalen Spaltung, ist zumindest in entwickelten Industrienationen, im Vergleich zum in der Wissensgesellschaft erforderlichen Erwerb einer entsprechenden Medienkompetenz, nahezu vernachlässigbar. Der annähernd flächendeckende Einzug von EDV-Systemen in den Büroalltag bestätigt dies. Liberalisierte Telekommunikationsmärkte und damit fallende Preise für den Internetzugang werden ihr Übriges zu dieser Entwicklung beitragen. Während die Nutzung des Computers als erweiterte Form der Schreibmaschine mittels der bereits erworbenen Kulturtechniken hinreichend gut bewältigt werden konnte, gilt dies für das Medium Internet jedoch nicht. Kubicek identifiziert ein Bündel bestehend aus vier verschiedenen Fähigkeiten, die es im Bezug auf das World Wide Web neu $\mathrm{zu}$ erlernen gilt, um dieses kompetent nutzen zu können (ebd.).

Erstens bedarf es der Fähigkeit, zwischen verschiedenen Informationsgattungen differenzieren zu können: Handelt es sich um amtliche Mitteilungen, politische Erklärungen oder Werbetexte. Zweitens muss der Nutzer in der Lage sein, sich im Medium Internet orientieren bzw. die angebotenen Indizierungsmethoden nutzen zu können. Drittens wird die Ausbildung eines Kriterienkataloges erforderlich, der eine kritische Bewertung der angebotenen Informationen ermöglicht. Viertens setzt eine aktive, d. h. sendende, Teilnehmerfunktion die Kompetenz zur Generierung von Inhalten voraus.

Es ist gerade die völlige Informationsfreiheit des Internets, die den Erwerb einer solchen Medienkompetenz erforderlich macht. Während in der Welt der Printmedien, des Fernsehens und des Radios Nachrichtenagenturen und Redaktionen Informationen vorstrukturieren und aufbereiten, sind derartige Filtermechanismen im World Wide Web kaum vorhanden. Hier stehen Millionen von Websites gleichberechtigt nebeneinander. Was einerseits eine Chance für die globale Informationsfreiheit ist, stellt sich gleichzeitig als enorme Herausforderung, bisweilen als Überforderung des Nutzers dar. Gibt ein Schüler bspw. einen Begriff wie „Holocaust" oder „Third Reich“ in eine internationale Suchmaschine ein, so wird er bereits unter den ersten zehn Fundstellen neben aufklärenden Seiten auch auf revisionistische bzw. rechtsextreme Inhalte stoßen (Siedschlag, Rogg, Welzel 2002, S. 102).

Derzeit ist es jedoch nahezu unmöglich, differenzierte Aussagen über die Medienkompetenz der Internetnutzer in Deutschland zu machen. Die verschiedenen Primärerhebungen zur digitalen Spaltung in Deutschland beschränken sich zumeist darauf, die Verbreitung von technischen Voraussetzungen zur passiven Partizipation abzufragen. Die vorliegenden Erhebungen zum Problemfeld Digital Divide sind daher vor allem für die Werbewirtschaft und werbefinanzierte Sektoren von Nutzen, da sie zielgruppenorientierte Aussagen über die Reichweite des Mediums zulassen. Dafür spricht auch eine Analyse der Auftraggeber der verschiedenen Erhebungen zur digitalen Spaltung in Deutschland. Anders als in den USA, wo die Soziodemographie der Internetnutzer durch öffentliche Stellen in regelmäßigen Zyklen erhoben wird (U.S. Department of Commerce 1995), werden entsprechende Studien hierzulande in den meisten Fällen von Unternehmen der Medien- sowie der IT-Branche in Auftrag gegeben und finanziert. ${ }^{4}$ Dieser Umstand mag auch erklären, weshalb diese Erhebungen die Internetnutzung zumeist erst ab dem 14. Lebensjahr erfassen - der unteren Grenze der werberelevanten Alterskohorte der 14- bis 29Jährigen - und quantitativ unbedeutendere Bevölkerungsgruppen, wie etwa Ausländer und/ oder Behinderte, die zudem speziellen sprachlichen bzw. physischen Barrieren ausgesetzt sind und daher besonders von der digitalen Spaltung betroffen sind, nicht berücksichtigen.

\section{Die ökonomische Verwertung von Wissen}

Besonders gravierende Auswirkungen auf die Chancengleichheit des Wissenszugangs könnte jedoch eine Thematik entfalten, die bislang in der Debatte über Digital Divide vernachlässigt wurde. Die diversen Analysen der digitalen Kluft und die darauf aufbauenden politischen Handlungsempfehlungen gehen zumeist davon aus, dass eine möglichst flächendeckende Versorgung aller Bevölkerungsgruppen mit Internetzugängen geeignet ist, eine dauerhafte Spaltung der Gesellschaft in Onliner und Offliner zu verhindern und so die Voraussetzungen 
geschaffen werden können für eine annähernde Chancengleichheit in der Wissensgesellschaft. Dies impliziert jedoch die Annahme, dass das Wissen selbst frei verfügbar sei und der $\mathrm{Zu}-$ gang dazu nur ein rein technisches Problem darstelle, wobei unter Umständen noch bestimmte Grundkenntnisse im Umgang mit IuKTechnologien zu vermitteln seien.

Diese Annahme steht jedoch im Widerspruch zu real beobachtbaren Ökonomisierungsstrategien in der digitalen Welt. Die Privatisierung von Wissen - auch als Venterisierung $^{5}$ bezeichnet (Kuhlen 2002a) - und deren ökonomische Verwertung nimmt eine Vielzahl von Formen an und es stellt sich die Frage, welche Auswirkungen dies auf die sich formierende Wissens- bzw. Informationsgesellschaft zeitigen wird.

Die Etablierung eines Property Rights Regimes soll in der Internetökonomie nicht zuletzt durch Digital Rights Management (DRM)Verfahren ermöglicht werden. DRM auf Softbzw. Hardware-Ebene dient der Kontrolle des gesamten Vertriebswegs digitalisierter Produkte und ermöglicht so die exakt skalierbare Festlegung, wie und in welchem Umfang digitale Informationen genutzt, gespeichert und vervielfältigt werden können. Potenzielle Anwendungsfelder von DRM sind somit neben Musik und Filmen gerade auch so genannte EBooks und E-Journals.

Neben Digital Rights Management zeichnen sich noch eine Reihe weiterer Verwertungsstrategien in der Internetökonomie ab:

- „Anfütterung“ der Nutzer: Nach einer Gewöhnungsphase der kostenlosen Bereitstellung digitalisierter Informationen werden diese in kostenpflichtige Inhalte umgewandelt. Verschiedene wissenschaftliche Verlage haben anfangs Bibliotheken, die ein bestimmtes Kontingent an Abonnements bezogen, kostenlosen Zugriff auf die OnlineVersionen ihrer Publikationen gewährt und diesen Service später mit zusätzlichen Gebühren versehen.

- Generierung technischer Pfadabhängigkeiten: Preisverbilligte oder kostenlose Produkte werden an bestimmte Zielgruppen abgegeben, häufig in Kombination mit der Erhebung von Nutzerinformationen über die Abnehmer. So geben zahlreiche große Softwareunternehmen ihre Produkte bspw. an Lehr- kräfte und Studenten verbilligt ab. So gelingt es, im beruflichen bzw. wissenschaftlichen Umfeld einen „Multiplikatoreneffekt“ zu erzielen.

- „Follow the Free“: Informationen und Dienstleistungen werden kostenlos angeboten und Umsätze werden durch den Verkauf von Komplementärprodukten oder weiterführenden Informationen erwirtschaftet. So bieten z. B. eine Reihe von Tageszeitungen und Zeitschriften einen Teil ihrer PrintAusgaben kostenlos im Netz an, während die erweiterte Nutzung, etwa der Archive älterer Ausgaben, kostenpflichtig ist.

- Die Nutzung des Nutzers: Dies geschieht u. a. durch die Sammlung von Informationen über den Nachfrager von digitalisierten Produkten und den Handel mit Nutzerprofilen. Technisch realisiert wird dies durch den Einsatz von Zwangsregistrierungen beim Abruf von digitalem Wissen, durch die Verwendung von Cookies oder so genannter SpyWare. So war etwa die Musiktauschbörse Napster, der eine Art Pionierrolle in der Free-Information-Bewegung zukam, auch bekannt dafür, dass bei der Installation des Programms ohne Wissen des Nutzers eine Reihe von Ausspäh-Modulen auf dem Rechner implementiert wurden.

- MicroPayment: Dieses kreditkartenlose Zahlungsverfahren soll zur Überwindung der Skepsis der Internetnutzer bei OnlineGeschäften und zur Akzeptanzbildung bei der Bezahlung kleiner Beträge für einzelne „Informationshäppchen“ beitragen, wie sie etwa beim Download der aktuellen TitelStory von Nachrichtenmagazinen anfallen.

Die Gesetzgebung auf internationaler sowie nationaler Ebene befindet sich derzeit noch in der Entwicklungsphase, ebenso muss die Rechtsprechung noch zu einem entsprechenden Modus Operandi finden. Gleichwohl zeichnet sich $\mathrm{ab}$, dass nicht zuletzt die Anbieter von DRM-Systemen, zu denen mittlerweile eine Vielzahl der weltweit operierenden Player der IT-Branche zählen, hierbei für sie nützliche rechtliche Rahmenbedingungen vorfinden werden. Besonders weit gediehen ist die U.S.amerikanische Gesetzgebung. Mit dem Digital Millenium Copyright Act (DMCA) wurden bereits Fakten geschaffen und die Entwicklung, Nutzung und Verbreitung von Software unter 
Strafe gestellt, die geeignet ist, CopyrightAnsprüche zu unterlaufen. Erfolgreiche Klagen wurden bereits geführt - bspw. gegen die Entwicklung und Distribution von Systemen zur Überwindung von DRM-Restriktionen bei elektronischen Büchern. Auf europäischer Ebene wurde die EU-Richtlinie zur Harmonisierung bestimmter Aspekte des Urheberrechts und verwandter Schutzrechte verabschiedet, um europaweit für eine Umsetzung der internationalen WIPO-Verträge zu sorgen. ${ }^{6}$

Das Bundesministerium der Justiz hat mittlerweile den Regierungsentwurf eines Gesetzes zur Regelung des Urheberrechts in der Informationsgesellschaft fertig gestellt, um die EU-Richtlinie rechtzeitig in nationales Recht umzusetzen. Der Informationswissenschaftler Kuhlen interpretiert den vorangegangenen Referentenentwurf, der sich eng an der Richtlinie orientierte, als „Verstärkung der exklusiven Verwertungsrechte der Urheber (wobei analog zur EU-Richtlinie eigentlich immer die Verwerter gemeint sind, wenn akzeptanzsichernd von ,Urhebern' die Rede ist)“ (Kuhlen 2002b). Zwar weise der Gesetzentwurf so genannte „Schrankenbestimmungen“ auf, die Urheberrechtsansprüche durch privates bzw. öffentliches Interesse an einer möglichst freizügigen Nutzung von Wissen einschränken sollten, doch bestünde die Gefahr, dass diese Schrankenbestimmungen durch die Verwendung so genannter "technisch wirksamer Maßnahmen“ umgangen werden könnten, wobei darunter nicht zuletzt DRM-Lösungen verstanden werden könnten.

\section{Von der sozialen zur digitalen Frage?}

Bei allen gut gemeinten Initiativen, die digitale Spaltung innerhalb der deutschen Gesellschaft zu überwinden, besteht die Gefahr, dass durch die rechtliche Verankerung eines Property Rights Regimes die Spaltung nicht aufgehen wird, sondern im Gegenteil eine neue Qualität bekommt. Impliziert der Terminus "Digital Divide" und die Debatte, die darüber geführt wird, dass es, rein normativ gesprochen, so etwas wie eine "digitale Gerechtigkeit" gibt, die beinhaltet, dass jeder Mensch, ungeachtet seiner Herkunft, Ausbildung oder seines Einkommens ungehinderten Zugang zum Netz und damit zu Information haben sollte, werden mit Bewegungen wie
DRM und ihrer Manifestierung - wie z. B. mit der Reform des Urheberrechts - Fakten geschaffen, die die Grenzen zwischen Onlinern und Offlinern verhärten können.

Die Ursache dafür liegt jedoch weniger im Internet selbst. Vielmehr reproduzieren die Phänomene Digitale Spaltung und Property Rights Regime traditionelle gesellschaftliche Trennlinien. So ist es kein Zufall, dass die Kategorie der Einkommensschwachen bei der Nutzung des Internets stark unterrepräsentiert ist, oder dass die empirischen Erhebungen über den Internetzugang der Bevölkerung gesellschaftliche Randgruppen wie Arbeitslose, Ausländer oder Behinderte eher außer Acht lassen.

Folglich werden auch Maßnahmen wie „Schulen ans Netz" oder „Initiative D21" die grundlegende gesellschaftliche Spaltung nicht überwinden. Wie die alarmierenden Ergebnisse der PISA-Studie der OECD vor Augen geführt haben, wird auch die rein quantitative Einführung moderner IuK-Medien keine stabile Basis für die Zukunft der deutschen Wissens- und Informationsgesellschaft schaffen. Viel mehr wird es hier, wie oben bereits angedeutet, auf die Qualität der Nutzung bzw. auf das qualitative Filtern und Selektieren von Informationen ankommen. Das setzt jedoch eine schulische Vermittlung über den Umgang mit den neuen Medien voraus und vor allem eine Anleitung zum Selbstlernen. Denn die rasante Eigendynamik dieser Technologien macht das einmal Erlernte in kürzester Zeit obsolet und erfordert ein kontinuierliches Lernen im Prozess ihrer Anwendung.

\section{Internetökonomie ohne Institutionen}

$\mathrm{Ob}$ denn die so genannte Wissens- und Informationsgesellschaft in Zukunft einen so großen Anteil am Bruttosozialprodukt haben wird, dass sie die traditionellen Industrien in der Bundesrepublik überholen wird, kann hier nicht beantwortet werden. Tatsache ist allerdings, dass sich eine "Internetökonomie" zu etablieren versucht, die es mit Herausforderungen aufnehmen muss, für die es auch aus anderen neuen Industriezweigen keine Best-practice-Modelle gibt. Die "Internetökonomie", und hier besonders die Digital Content Industrie, hat nach einer euphorischen und zum Teil materiell unbegründeten Boomphase mehrere bittere Konsolidierungs 
prozesse durchstehen müssen. Selbst Unternehmen, die an der Börse hoch gehandelt wurden, mussten starke Einbußen oder sogar den Bankrott erleben. In der gegenwärtig noch andauernden Konsolidierungsphase wird vor allem deutlich, dass die Geschäftsmodelle der dot.coms auf instabilen Füßen standen, was z. T auch auf ein fehlendes, langfristig gewachsenes Institutionengefüge und auf eine mangelnde rechtliche Absicherung der Verwertungsrechte digitaler Inhalte zurückzuführen ist. Vor diesem Hintergrund ist es nicht verwunderlich, dass das Digital Rights Management vehemente Verfechter findet. Gleichzeitig entsteht hier eine neue Industrie, um mittels neuer technischer Lösungen das unautorisierte Kopieren und Weitergeben von digitalen Inhalten zu verhindern.

Innerhalb der Internetökonomie muss stark differenziert werden zwischen den einzelnen Anbietern. Dass der Tauscheuphorie von $\mathrm{Mu}-$ sik- und Videoartikeln im Internet inzwischen rechtliche und technische Grenzen gesetzt sind, werden selbst die vehementesten Verfechter des Free Content nachsehen. Schließlich ist auch der analoge Tausch strukturell eingeschränkt und sei es durch die Qualität der Kopien. ${ }^{7}$

\section{Digitales Recht und digitale Gerechtig- keit - ein Widerspruch?}

Wie die vorherigen Ausführungen zeigen, ist die Entwicklung der Internetökonomie und ihrer institutionellen Absicherung durchaus im Zusammenhang mit der Debatte um die Digitale Spaltung zu sehen. Daraus ergeben sich weitere wissenschaftliche Forschungsfelder um einzuschätzen, entlang welcher gesellschaftlicher Linien der Einsatz von IuK-Technologien die traditionelle gesellschaftliche Spaltung untermauert bzw. verstärkt, und welcher politische Handlungsbedarf sich daraus ergibt, um langfristig den Weg in die Wissensökonomie einzuleiten, an der nicht nur einzelne Akteursgruppen beteiligt sein sollen. Um Digitales Recht und Digitale Gerechtigkeit nicht zu einer contradictio in adjectio werden zu lassen, müssen politische und wirtschaftliche Akteure ebenso in den politischen Entscheidungsfindungsprozess integriert werden wie (potenzielle) Nutzer. Der Versuch der Überwindung der digitalen Spaltung und der Etablierung institutioneller Strukturen für die Geschäftsstrategien von Digital Content Firmen sollte nicht allein auf kurzfristige wirtschaftliche Effekte abzielen. Erforderlich ist viel mehr eine weitreichende Strategie, um den langfristigen volkswirtschaftlichen Mehrwert für die Zukunft der Wissens- und Informationsgesellschaft zu erhöhen. Dies kann mitunter besser geschehen, wenn Information und Wissen einen hohen Diffusionsgrad erreichen.

\section{Anmerkungen}

1) http://www.initiatived21.de/

2) http://www.san-ev.de/

3) Eine vergleichbare Formulierung findet sich auch in Artikel 19 der allgemeinen Erklärung der Menschenrechte: Everyone has the right to freedom of opinion and expression; this right includes freedom to hold opinions without interference and to seek, receive and impart information and ideas through any media and regardless of frontiers.

4) Die im Juli 1995 in den USA veröffentlichte Studie „Falling through the net“ untersuchte erstmals systematisch die Durchdringung privater Haushalte mit Internetzugängen. Dabei wurden Ungleichverteilungen im Hinblick auf die Variablen Geschlecht, Einkommen, Wohnort, Alter, Bildung sowie ethnische Zugehörigkeit erfasst. Diese in Kooperation von National Telecommunications and Information Administration (NTIA) und U.S. Census Bureau durchgeführte Erhebung wurde mittlerweile zum vierten Mal aktualisiert.

5) Unfreiwilliger Namensgeber des als Antonym zur Napsterisierung konzipierten Begriffs der „Venterisierung“ ist der U.S.-Amerikaner Craig Venter, der mit seinem Unternehmen Celera Genomics die privatwirtschaftliche Verwertung von Informationen über menschliches und tierisches Genom betreibt, welche teilweise mit öffentlichen Mitteln erforscht wurden.

6) Dies ist zum einen der Urheberrechtsvertrag der WIPO (World Intellectual Property Organisation) sowie zum anderen der WIPO-Vertrag über Darbietungen und Tonträger.

7) Komplexer wird die Entwicklung jedoch z. B. im Wissenschaftsbetrieb, d. h. bei den wissenschaftlichen Verlagen.

\section{Literatur}

FAZ 15.09.2002: Die Wahrheit kostet heute 13 Euro 50

GfK - Gesellschaft für Konsumforschung, 2001: GfK Online-Monitor. http://www.gfk.de/produkte/eigene pdf/online_monitor.pdf [Download: 26.09.2002] 
Hauser, H., 2001: Die Bedeutung von UN-Organisationen bei der Förderung und Regulierung des elektronischen Handels. In: TA-Datenbank-Nachrichten, Nr. 4, 10. Jahrgang; S. 11-21

Hochreiter, R., 2001: Die E-Commerce-Politik der Bundesregierung. In: TA-Datenbank-Nachrichten, Nr. 4, 10. Jahrgang; S. 50-56

Initiative D21, 1999: Memorandum. http://www. initiatived21.de/profil/ueber/memorandum-d21.pdf [Download: 26.09.2002]

Kubicek, H., 1999: Vor einer Teilung der Gesellschaft: Chancengleichheit und Multimedia. Potsdamer Fachkongress „Chancengleichheit - Leitbegriff für Politik und Gesellschaft im 21. Jahrhundert", 12. November 1999. http://www.digitale-chancen.de/transfer/downloads/MD46.pdf

[Download: 14.04.2002]

Kuhlen, R., 2001: Universal Access - Wem gehört das Wissen? http://www.inf-wiss.uni-konstanz.de/ People/RK/Vortraege01-Web/publikationstext.pdf [Download: 26.09.2002]

Kuhlen, R., 2002a: Napsterisierung und Venterisierung. Bausteine zu einer politischen Ökonomie des Wissens. In: PROKLA. Zeitschrift für kritische Sozialwissenschaft, Heft 126, Nr. 1, 32. Jahrgang, S. $57-88$

Kuhlen, R., 2002b: Stellungnahme zum Referentenentwurf des Justizministeriums zur Umsetzung der EU Richtlinie 2001/29/EG. http://www.nethics.net/ nethics/de/themen/urheberrecht/stellungnahme zum eu_referentenentwurf.html [Download 30.09.2002]

$O E C D$, 2001: Understanding the Digital Divide. Paris: OECD. http://www.oecd.org/pdf/M00002000/ M00002444.pdf [Download: 26.09.2002]

Siedschlag, A.; Rogg, A.; Welzel, C., 2002: Digitale Demokratie. Willensbildung und Partizipation per Internet. Opladen: Leske + Budrich.

U.S. Department of Commerce, 1995: Falling through the net. A survey of the "Have Nots" in Rural and Urban America. http://www.ntia.doc.gov/ ntiahome/fallingthru.html [Download: 11.03.2002]

van Eimeren, B.; Gerhard, H., 2002: ARD/ZDFOnline-Studie 2002. Entwicklung der OnlineNutzung in Deutschland: Mehr Routine, weniger Entdeckerfreude. In: Media Perspektiven 8/2002, S. 346-362.

\section{Kontakt}

Dr. Susanne Giesecke

David Linse

VDI/VDE-Technologiezentrum

Informationstechnik $\mathrm{GmbH}$

Rheinstr. 10 B, 14513 Teltow

Tel.: +49 (0) 3328 / 435 - 166
Fax: +49 (0) 3328 / 435 - 216

E-Mail: ita@vdivde-it.de

Internet: http://www.innovationsanalysen.de/

\section{$《 \gg$}

